\title{
A true new gene in ophiocomina nigra: an ophuirid Igkappa gene
}

\begin{abstract}
An Igkappa gene was discovered in the transcriptome of the Ophuirid: Ophiocomina nigra. So, with the Asterid: Asterias rubens, 2 classes of Echinodermata, possess each an Igkappa gene. It remains enigmatic, when we have a look on the 3 other classes without this gene: The Echinids, Holothurids, and Crinoids
\end{abstract}

Keywords: invertebrate, ophiocomina nigra, ophuirid igkappa gene, HRP

\author{
Volume 5 Issue I - 2018 \\ Michel Leclerc, ',2,3 Yannick Marie, 'Dominique \\ Davoult, ${ }^{2}$ Ariane Jolly, ${ }^{3}$ Pierre de la Grange ${ }^{3}$ \\ 'Hôpital de la Salpêtrière, France \\ 2Biologic Station of Roscoff, University Pierre et Marie Curie, \\ France \\ ${ }^{3}$ ICM Genosplice, France
}

Correspondence: Michel Leclerc, ICM, Hôpital de la Salpêtrière, 556 rue Isabelle Romée, 45640 Sandillon, France, Email mleclerc45@gmail.com

Received: January 09, 2018| Published: January 29, 2018

\section{Introduction}

Recently, investigations performed in our laboratory, have provided evidence, that the Ophuirid: Ophiocomina nigra (Echinodermata), presented antibody-like reactions with cellular and humoral reactions to peroxydase antigen. These reactions are similar to those observed in the sea star Asterias rubens, another Echinodermata ${ }^{1}$ and we know that the Asterias rubens genome contains the sea star Igkappa gene with Ig sites, ${ }^{2}$ a Fab gene, a Fc receptor gene. The aim of this work consists to explore immune genes in the genome of virgin Ophiocomina nigra.

\section{Materials and methods}

Ophiocomina nigra was collected to the Biologic station of Roscoff (France). Digestive coeca were excised and treated with Uptizol (Interchim) to obtain m RNA Ophiocomina nigra.

\section{Preparation of library (RNA), by the use of the kappa mRNA hyper prep kit}

Sequencing: the sequencing was done with a NextSEq 500 Illumina( 2.75 bases). Transcriptome was assembled from RNA-Seq fastq files using Trinity v2.1.1 ${ }^{3}$ with default parameters. A BLAST database was created with the assembled transcripts using makeblastdb application from ncbi-blast $+(\mathrm{v} 2.2 .31+)$. The sequences of transcripts of interest were then blasted against this database using blastn application from ncbi-blast $+^{4}$ ) with parameter word size 7 .

\section{Results}

An obtained blast against homo sapiens was very highly significant (E-value of 2,00E-12). Undoubtly it brings the evidence of the existence of an Ophuirid-IGKappa gene (Table 1).

\section{The sequence follows}

>BC030813.1 Homo sapiens immunoglobulin kappa locus, mRNA (cDNA clone MGC:22645 IMAGE:4700961), complete cds 5 ' G A G G A A C T G C T C A G T T A G G A C C C A G A C G G A A C C AT G GAAG C C C CAGCG CAG C T T C T C T T C C T C C T G C TA C T C T G G C T C C C A GA TA C C A C T GGAGAAATAG T GAT GACGCAGTCT C CAG C C A C C C T G T C T G T G T C T C C A G G G GA A A G A G C C A C C C T C T C C T G C A G G G C C A G T C A G A GT GTTAC CAGCAACT TAGC CTGGTACCAGCAGACA C C T G G G C A G T C T C C C A G G C T C G T C A T C TA T G G T G C AT C CAGCAGGGCCAGTGGTGTCCCAGCCAGGTTCA G T G G C A G T G G G T C T G G GA CA GA G T T C A C T C T C A C CATCAGCAGCCTGCAGTCTGAAGAT T T T GCAGT T TA T TAC T G T CA G C A G TATAATAAG T G G C C G C A C A C T T T T GGC CAGGGGACCAAGCTGGACATCAAACGAAC T G T G G C T G C A C C A T C T G T C T T C A T C T T C C C G C C A T C T GAT GAGCAGT T GAAAT C T GGAAC T GC C T C T G T T G T G T G C C T G C T G A A T A A C T T C TA T C C C A G G GA G G C CAAAGTACAGTGGAAGGTGGATAACGCC C T C CAATC GGGTAAC T C C C A G GA GAGTGTCACAGA G C A G GA C A G C A A G GACAG CAC C TA CA G C C T CA G C A G C A C C C T G A C G C T GA G C A A A G C G A C TAC GAGAAACACAAAG T C TA C G C C T G C GAAG T C A C C C A T C A G G G C C T GA G C T C G C C C G T C A C A A A G A G C T T CA A C A GG G A GAG T G T TAGAGGGAGAAGTGCC C C CAC C T G C T C C T C AGT T C C A G C C T GA C C C C C T C C C A T C C T T T G G C C T C T GA C C C T T T T T C CACAGGGGAC C TAC C C C TAT TG C GG T C C T C C A G C T C A T C T T T C A C C T C A C C C C C C T C C T C C T C C T T G G C T T TAA T TA T G C TAA T G T T G G A G G A GA A T G AATAAATAAAGTGAATCTTTGCAAAAAA A A A A A A A A A A A A A A A A A A A A A A A A A A A A A A A A A A A A A A A A A A AAAAAAAAAAAAAAAAAAAAAAAAAAAAAAAAAAAAAAA 3'

Table I An obtained blast against homo sapiens was very highly significant (E-value of 2,00E-I2)

\begin{tabular}{llllllll}
\hline Query ID & $\begin{array}{l}\text { Query } \\
\text { name }\end{array}$ & Subject ID & Identity & Length & Mismatch & Gapopen & E-value \\
\hline BC0308I3.I & Igk & TRINITY_DN64572_C0_gl_il & 89.47 & 57 & 6 & 0 & $2,00 E-12$ \\
\hline
\end{tabular}




\section{Conclusion and discussion}

A gene of about 960 nucleotides appears. It is longer than the one found in Asterias rubens (Asterid) in immunized sea stars to HRP. ${ }^{2}$ For the second time, "The classic immunology" is broken with the emergence, in Invertebrates, of a gene which has the property of Invertebrate primitive antibody. But it would be necessary to correlate this gene to the obtained immune reaction in Ophuirids. A bright avenir is opened in the field of comparative immunology.

\section{Highlights}

- In 2015 discovery of the sea star Igkappa gene

- Discovery of the sea star Primitive antibody

\section{Acknowledgements}

None.

\section{Conflict of interest}

The author declares no conflict of interest.

\section{References}

1. Leclerc M, Brillouet C. Evidence of antibody-like substances secreted by axial organ cells of the starfish Asterias rubens. Immunology Letters. 1981;2(5-6):279-281.

2. Nadine Vincent, Magne Osteras, Patricia Otten, et al. A new gene in $A$. rubens: A sea star Ig kappa gene. Meta Gene. 2014;2:320-322.

3. Manfred G Grabherr, Brian J Haas, Moran Yassour, et al. Full-length transcriptome assembly from RNA-Seq data without a reference genome. Nature Biotechnology. 2011;29:644-652.

4. Stephen F Altschul, Warren Gish, et al. Basic local alignment search tool. Journal of Molecular Biology. 1990;215(3):403-410. 